Research Paper

\title{
The Efficacy and Toxicity of Lobaplatin-contained Chemotherapy in Extensive-stage Small-cell Lung Cancer
}

\author{
Ning-ning Zhou ${ }^{* 1}$, Yuan-yuan Zhao ${ }^{* 1}$, Lin-zhu Zhai ${ }^{* 2}$, Chao-mei Ruan³, Yun-peng Yang1, Yan Huang1, Xue \\ Hou $^{1}$, Li-kun Chen ${ }^{1}$, Ting Zhou ${ }^{1}$, Li Zhang ${ }^{1 凶}$ \\ 1. Department of Medical Oncology, Sun Yat-Sen University Cancer Center, State Key Laboratory of Oncology in South China; Collaborative Innovation \\ Center for Cancer Medicine, Guangzhou, 510060, PR China. \\ 2. Department of Medical Oncology, The First Affiliated Hospital of Guangzhou University of Chinese Medicine, Guangzhou 510405, PR China. \\ 3. Medical imaging department, Sun Yat-Sen University Cancer Center, State Key Laboratory of Oncology in South China; Collaborative Innovation Center \\ for Cancer Medicine, Guangzhou, 510060, PR China. \\ * These authors contributed equally to this work. \\ $\triangle$ Corresponding author: Li Zhang (email: zhangli@sysucc.org.cn)
}

(c) Ivyspring International Publisher. This is an open access article distributed under the terms of the Creative Commons Attribution (CC BY-NC) license (https://creativecommons.org/licenses/by-nc/4.0/). See http://ivyspring.com/terms for full terms and conditions.

Received: 2017.12.25; Accepted: 2018.03.31; Published: 2018.06.05

\begin{abstract}
To assess the efficacy and toxicity of Lobaplatin (LBP) -contained chemotherapy on extensive stage small-cell lung cancer (ES-SCLC), we conducted a prospective, single-arm, and multicenter Phase IV clinical trial on Lobaplatin (ChiCTR-ONC-13003471), and used the patient clinical data obtained from our cancer center to perform the analysis. Previously untreated patients with ES-SCLC were given LBP intravenously (IV) at 30 $\mathrm{mg} / \mathrm{m}^{2}$ on day 1 and etoposide IV at $100 \mathrm{mg} / \mathrm{m}^{2}$ on day 1,2 , and 3 . The treatment was cycled every 21 days, lasting for four to six cycles. The patients with second-line treatment or above were also included in the study, and they were treated with LBP-contained regimen: a single dose of LBP at $50 \mathrm{mg} / \mathrm{m}^{2}$ on day 1 through IV; combined application, LBP30 mg/m² IV on day 1. From May 2015 to August 2016, 36 patients were enrolled in the study at our cancer center. For the 30 first-line patients, the median overall survival (OS) and the median progression-free survival (PFS) was 13.0 months (ranging from 11.2 to 14.7 months) and 4.7 months (ranging from 1.6 to 7.7 months) respectively, with overall response rate of $57 \%$ and disease control rate of $85.7 \%$. For the 6 patients with second-line treatment or above, one patient got a partial response (PR) and four patients got a stable disease (SD). The most frequent drug-related adverse effects were leukopenia and neutropenia, and no grade 3/4 hepatotoxicity or nephrotoxicity was observed. These results indicated that LBP-contained chemotherapy was effective and tolerable for extensive stage SCLC in terms of response and survival. However, due to the small sample size of this study, we need to wait for the OS data of phase III clinical trial and the final data of this multicenter Phase IV study to draw the conclusion.
\end{abstract}

Key words: Small cell lung cancer; Lobaplatin; Chemotherapy

\section{Introduction}

Lung cancer is one of the most common cancers in the world. It is the leading cause of death in China, with increasing incidence and death rate $[1,2]$. Small cell lung cancer represents $15-20 \%$ of the newly diagnosed lung cancer around the world, and is also the most aggressive subtype [3]. As reported, the median survival of SCLC without treatment is only 2-4 months. About two-thirds of the SCLC patients present to clinic with obvious metastasis, which is classified as extensive stage SCLC (ES-SCLC) [4].
The recommended treatment for untreated ES-SCLC patients is a combination chemotherapy of platinum-based drug and etoposide, which has been the standard regimen since mid-1980s [5, 6]. Although this chemotherapy produces a good response rate at $60 \%-70 \%$ for ES-SCLC, all the patients experience relapses, with a median survival of 9.3-12.8 months. Several attempts have been made to improve the efficacy, including alternating non-cross-resistant drugs, increasing total dose and dose intensity, or 
adding treatment courses and number of drugs, but none of them led to improved survival [7].

As a common platinum therapy, cisplatin treatment frequently causes severe toxicities, such as gastrointestinal toxicity, nephrotoxicity and neurotoxicity. Lobaplatin is a third-generation platinum derivative, which has been approved in China for treating metastatic breast cancer, chronic myelogenous leukemia and SCLC (http://app2.sfda .gov.cn/datasearchp/index1.do?tableId $=25 \&$ tableNa me=TABLE25\&scdw=scdw\&Id=182332). It has showed promising activities in several preclinical studies, with incomplete cross-resistance with other platinum drugs, and lower renal toxicity compared to cisplatin [8]. Our cancer center has participated in the prospective, single-arm and multicenter Phase IV clinical study to assess the safety and efficacy of Lobaplatin-contained chemotherapy for small cell lung cancer. The clinical study has registered in Chinese Clinical Trial Registry (ChiCTRONC-13003471). In this paper, we prospectively assessed the efficacy and toxicities of Lobaplatin-contained chemotherapy for ES-SCLC using the clinical data obtained from our cancer center.

\section{Materials and methods}

\section{Patient inclusion and exclusion criteria}

Inclusion criteria: Age more than 18 years old; confirmation of ES-SCLC by pathological or cytology tests (extensive stage is required in our cancer center); at least one measurable lesion; the Eastern Cooperative Oncology Group (ECOG) performance status ranging from 0 to 2; at least 3 months of life expectancy; with sufficient organ functions: absolute neutrophil count $\geq 1.5 \times 109 / \mathrm{L}$, platelet count $\geq 100 \times 109 / \mathrm{L}$, hemoglobin $\geq 90 \mathrm{~g} / \mathrm{L}$, serum total bilirubin $\leq 1.5 \times$ the upper limit of normal (ULN), serum ALT and AST $\leq 2.5 \times$ ULN $(\leq 5 \times \mathrm{ULN}$ if patients had liver problems), serum creatinine $\leq \mathrm{ULN}$, Creatinine clearance (Ccr) $\geq 60 \mathrm{ml} / \mathrm{min}$ (CockcroftGault); under contraception during and 6 months post treatment; not under breastfeeding; voluntarily participated and signed informed consent document.

Exclusion criteria: Allergic to platinum drugs used in the trial; with coagulation dysfunction; not meeting inclusion criteria for any reasons.

\section{Study design}

Previously untreated ES-ECLC patients were treated with LBP intravenously (IV) at $30 \mathrm{mg} / \mathrm{m}^{2}$ on day 1 , and etoposide IV at $100 \mathrm{mg} / \mathrm{m}^{2}$ on day 1,2 , and 3 . The treatment was cycled every 21 days, lasting for four to six cycles. Granulocyte colony stimulating factors were allowed to use at cycle 2 and beyond if the patients developed febrile neutropenia. The patients with second-line treatment or above were also included in the study. The specific Lobaplatincontained regimen for these patients was determined by researchers: Using single drug, LBP50 $\mathrm{mg} / \mathrm{m}^{2}$ intravenously (IV) on day 1; combined application, LBP30 $\mathrm{mg} / \mathrm{m}^{2}$ intravenously (IV) on day 1 .

\section{Toxicity and response evaluation}

Before the start of every treatment cycle, blood cell count, urinalysis, and biochemistry tests were performed to assess renal and hepatic functions, as well as electrolytes. Toxicity was graded based on the National Cancer Institute Common Terminology Criteria for adverse events version 3.0 (http://ctep.cancer.gov/protocolDevelopment/electr onic_applications/docs/ctcaev3.pdf). To measure the tumor sizes and locations, and to assess the responses, radiographic studies including computerized tomography (CT) scan were performed within 4 weeks prior to the treatment, as well as after every two cycles of treatment. All the response evaluation was based on the Response Evaluation Criteria in Solid Tumors (RECIST) [9].

\section{Dose modifications for toxicity}

For patients with grade 3 febrile neutropenia and grade 4 hematologic toxic effects, the doses of etoposide and LBP in subsequent cycles were reduced to $80 \%$ of the original doses. In addition, treatment was postponed until neutrophil count $\geq 1.0 \times 109 / \mathrm{L}$, platelet count $\geq 85 \times 109 / \mathrm{L}$, hemoglobin $\geq 85 \mathrm{~g} / \mathrm{L}$, and creatinine clearance $(\mathrm{Ccr}) \geq 60 \mathrm{ml} / \mathrm{min}$ (CockcroftGault). The patients with therapy delay caused by toxicity for more than 2 weeks were removed from the study.

\section{Tumor marker assessment}

The serum tumor markers (TM) of lung cancer, including pro-gastrin-releasing peptide (Pro-GRP), neuron-specific enolase (NSE), carcino-embryonic antigen (CEA), cytokeratin 19 fragment (Cyfra21-1), cancer antigen125 (CA125), and cancer antigen153 (CA153) were tested at baseline and before each treatment cycle. The tests were performed in the clinical laboratory of our cancer center.

\section{Statistical analysis}

An intention-to-treat (ITT) analysis was used to evaluate the efficacy and safety of the treatment. PFS was defined as the time interval between the date of registration and the date of progression/death, or the last known date of progression free (censored). OS was defined as the time interval between the date of registration and the date of death from any causes, or the last date of follow-up (censored). PFS and OS were 
plotted and calculated using the Kaplan-Meier method. All analyses were carried out using SPSS software version 22.0 (SPSS Statistics, IBM, and New York, NY).

\section{Ethics approval}

The study protocol was reviewed and approved by the ethics committee of Sun Yat-sen University Cancer Centre. The written informed consent was obtained from every study participant.

\section{Results}

\section{Patients}

Between May 2015 and August 2016, thirty-six patients were enrolled in the study at our cancer center. All patients received the treatment as planned and were assessed in intent-to-treat (ITT) analysis for toxicity, response, and survival measurements. The clinical characteristics of these patients were as follows: $83.3 \%$ male; $88.9 \%$ with ECOG PS ranging from 0 to 1 ; median age of 57.5 years old (ranging from 31 to 71 years old; Table 1 ).

Table 1. The baseline characteristics of the patients

\begin{tabular}{ll}
\hline Characteristics & No. of patients (\%) \\
\hline Gender & $30(83.3)$ \\
Male & $6(16.7)$ \\
Female & \\
Age (years) & 57.5 \\
Median & $(31.71)$ \\
Range & \\
Performance status (ECOG) & $32(88.9)$ \\
$0-1$ & $4(11.1)$ \\
2 & \\
LDH & $24(66.7)$ \\
$\leq$ ULN & $12(33.3)$ \\
$>$ ULN & \\
Smoking status & $8(22.2)$ \\
Non-smoker & $28(77.8)$ \\
smoker & $30(83.3)$ \\
Chemotherapy lines & $6(16.7)$ \\
First line & $13(36.1)$ \\
Second and more lines & $23(63.9)$ \\
Brain radiotherapy & $11(30.6)$ \\
Yes & $25(69.4)$ \\
No & \\
TRT & \\
Yes & \\
No & \\
\hline Abbreviations: LDH, lactate dehydrogenase; ULN, upper limit of normal; ECOG, \\
Eastern Cooperative Oncology Group; TRT, thorax radiotherapy \\
\end{tabular}

\section{Treatment delivery}

A total of 131 cycles of treatment were administered, with the median cycle number of 3.5 for all patients and 4 for the 30 first-line patients. 16 $(53.3 \%)$ out of the 30 first-line patients completed the entire 4-6 cycles of chemotherapy as scheduled. From all patients (36), 18 (50\%) had prolonged treatment; 3 received reduced doses, and 2 of them terminated the treatment because of severe neutropenia or anemia.

\section{Efficacy}

From the 30 first-line patients, 28 were eligible for response assessment. Objective tumor response was observed in 16 patients, and stable disease appeared in 8 patients, with the overall response rate of $57 \%$ and disease control rate of $85.7 \%$. Among the six patients with second-line treatment or above, one patient got a PR and four patients got a SD. By the time of survival assessment in Feb 2017, twenty patients had died. The median progression-free survival (PFS) for the 36 patients was 4.7 months (ranging from 2.8 to 6.5 months), and the median overall survival (OS) was 11.8 months (ranging from 8.0 to 15.6 months). The PFS and OS for the first-line patients were 4.7 months (ranging from 1.6 to 7.7 months) and 13.0 months (ranging from 11.2 to 14.7 months), respectively (Figure 1).

A
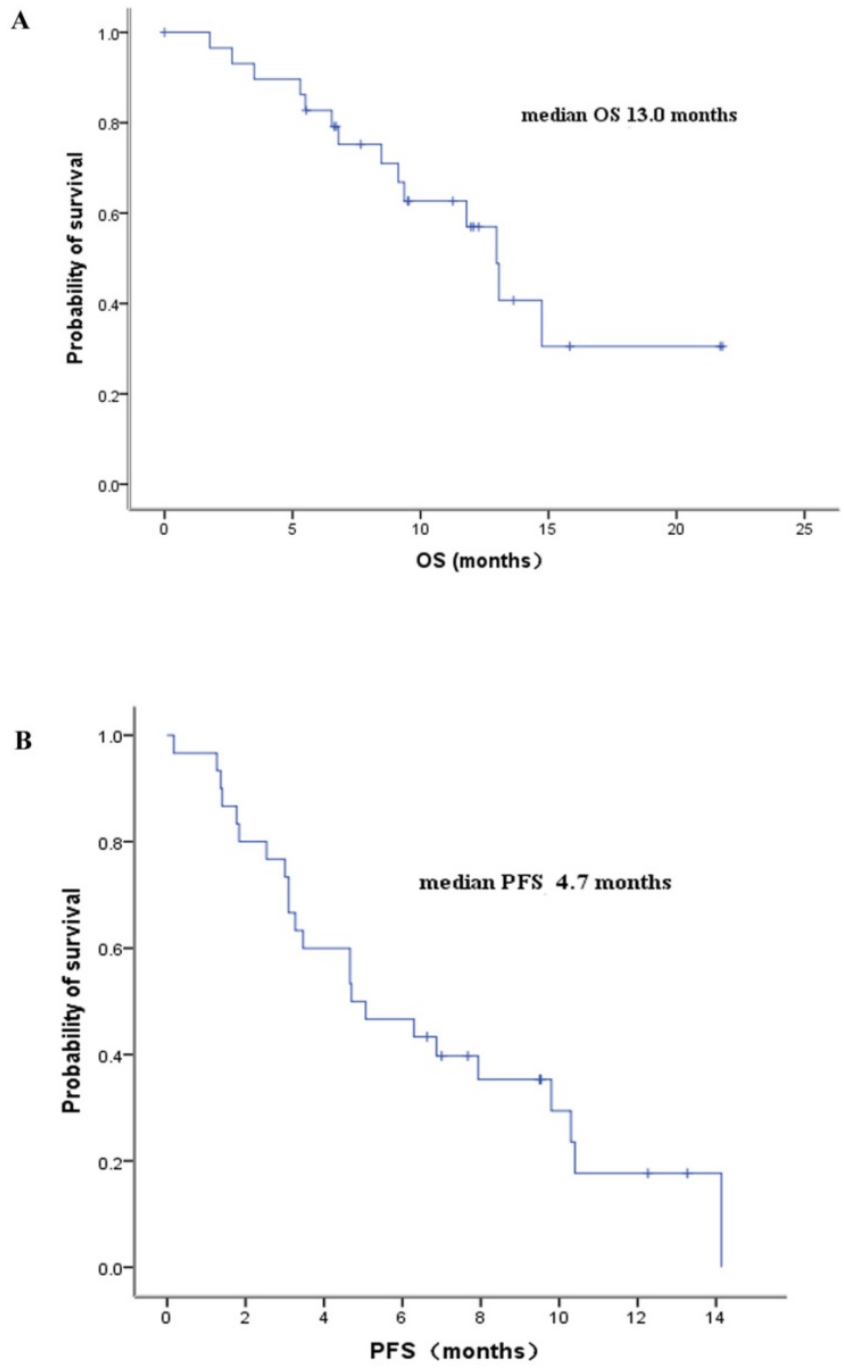

Figure 1. Kaplan-Meier survival curves of the ES-SCLC patients with Lobaplatin-contained first-line treatment. 


\section{Safety}

All the 36 patients were tested for toxicity. The most commonly observed toxicity was hematologic toxicity. Major toxicities with grade $\geq 3$ were listed in Table 2. Grade 3 or 4 leukopenia and neutropenia were observed in $55.5 \%$ and $58.3 \%$ of the patients, respectively. The patients with Grade 3 leukopenia or neutropenia were successfully treated with granulocyte colony-stimulating factor (G-CSF). In addition, $25-30 \%$ of the patients experienced Grade 3 or 4 thrombocytopenia and anemia. For two patients, the treatment was terminated due to severe neutropenia or anemia. No treatment-related death occurred in this study. The non-hematologic toxicities occurred in this study were all in grade 1 or 2 . No grade $3 / 4$ hepatotoxicity or nephrotoxicity was observed. Due to the preventive use of antiemetics during chemotherapy, most patients experienced mild nausea/vomiting.

Table 2. The adverse events with maximum severity (Grade 3/4) across all cycles of treatment

\begin{tabular}{lll}
\hline & $\begin{array}{l}\text { Frequency }(\%) \\
\text { all patients }(\mathrm{N}=36)\end{array}$ & $\begin{array}{l}\text { Frequency }(\%) \\
\text { first line patients }(\mathrm{N}=30)\end{array}$ \\
\hline $\begin{array}{l}\text { Toxicity (Grade 3/4) } \\
\text { Hematologic }\end{array}$ & & \\
$\begin{array}{l}\text { Neutropenia } \\
\text { Leukopenia }\end{array}$ & $21(58.3)$ & $17(56.7)$ \\
Thrombocytopenia & $20(55.6)$ & $17(56.7)$ \\
Anemia & $9(25.0)$ & $8(26.7)$ \\
Non-hematological & $10(27.8)$ & $9(30.0)$ \\
Hepatotoxicity & None & \\
Nephrotoxicity & None & None \\
Nausea/vomiting & None & None \\
\hline
\end{tabular}

\section{Serum tumor markers}

According to the test report from the clinical laboratory, the upper normal limits (UNL) of the test variables were: Pro-GRP 43ng/L, NSE $15.2 \mathrm{ng} / \mathrm{ml}$, CEA 5ng/ml, Cyfra21-1 3.3ng/ml, CA125 35U/ml, and CA153 25U/ml. We analyzed the tumor markers in 30 first-line patients, and found that the basal levels of Pro-GRP and NSE were elevated in more than $90 \%$ of the patients; Cyfra21-1 and CA153 were not changed or slightly elevated; CEA and CA125 were increased in $46 \%$ and $52 \%$ of the patients. These results suggested that Pro-GRP and NSE were the most sensitive indicators of small cell lung cancer.

\section{Discussion}

Studies have shown that Lobaplatin has various advantages as a drug, including potent antineoplastic activities, no significant nephrotoxicity or neurotoxicity [10], and no cross-resistance with cisplatin [11]. Preclinical study has demonstrated that Lobaplatin had significant antitumor activity against NSCLC both in vitro and in vivo [12]. In phase II clinical trials, Lobaplatin exhibited an active anti-tumor activity in multiple solid tumors, such as hepatocellular carcinoma [13], breast cancer [14], and metastatic nasopharyngeal cancer (NPC) $[15,16]$.

The outcomes of ES-SCLC patients are often dismal, with median overall survival less than 10 months and 5-year survival <5\% [17]. Currently, the choice of first-line treatment for ES-SCLC is four to six cycles of etoposide combined with a platinum salt (cisplatin or carboplatin), which results in a median survival of 8 to 10 months [18]. Combination chemotherapy of irinotecan and cisplatin for ES-SCLC leads to equal or better survival than etoposide and cisplatin, with a median survival of 9.3-12.8 months $[19,20]$. In our study, the median OS for the first-line patients was 13.0 months. To our best knowledge, this is the first overall survival data of Lobaplatin in SCLC. Our results demonstrated that the EL regimen was better than or equal to previous standard regimens in terms of OS. However, due to the small patient number in our study, we still need to wait for the final OS results of the phase III study on Lobaplatin and etoposide for ES-SCLC [21], as well as the final data of this multicenter Phase IV study.

In the previous phase III study of Lobaplatin and etoposide in ES-SCLC patients, the median PFS and the disease control rate (DCR) were 5.37 months and $82.64 \%$ in the first-line treatment [21], which is consistent with our results (median PFS of 4.7 months and disease control rate of $85.7 \%$ for the first-line patients).

Welink et al. reported that the dose-limiting toxicity of Lobaplatin was thrombocytopenia, with the threshold dose of $50 \mathrm{mg} / \mathrm{m}^{2}$ [22]. However, in our study, the most common hematological toxicities were leukopenia and neutropenia, which occurred in 17 patients with Grade 3/4 (56.6\%) in first-line treatment. The Lobaplatin dose used in our study was $30 \mathrm{mg} / \mathrm{m}^{2}$, which was different from the previous study and might lead to different side effects [22]. The toxicity profile of our study was consistent with another two Phase II studies [16, 23], which also showed that when the Lobaplatin dose was reduced to $30 \mathrm{mg} / \mathrm{m}^{2}$, the most common hematological toxicities were leukopenia and neutropenia. Moreover, no grade $3 / 4$ hepatotoxicity or nephrotoxicity was observed in our study. Therefore, our study suggested that Lobaplatin regimen could be well tolerated in SCLC patients.

In our study, the effects of Lobaplatin in second-line therapy or above were investigated in a small patient cohort. Among the six patients with second-line treatment or above, one patient got PR and four patients got SD. We found that the combined Lobaplatin regimen showed effect in the second line 
treatment with tolerable toxicity, which needs to be further verified. Tumor markers including Pro-GRP, NSE, Cyfra21-1, CA153, CEA and CA125 were evaluated in our study. As reported previously, NSE and Pro-GRP were useful markers for the diagnosis and therapeutic monitoring in SCLC patients [24, 25]. In our study, elevated Pro-GRP and NSE were detected in more than $90 \%$ of ES-SCLC patients before treatment, which confirmed the role of these two markers in SCLC diagnosis.

In summary, according to the results in our cancer center, Lobaplatin-contained chemotherapy was effective in terms of response and survival compared with other regimens for ES-SCLC. However, due to the small sample size, the OS data of phase III clinical study [21] and the final data of this multicenter Phase IV study are needed to draw the final conclusion.

\section{Acknowledgements}

This work was funded by the China National Natural Science Foundation (81502352 and 81501986), Guangzhou University of Chinese Medicine "High Level University Construction" Project (A1-AFD 018171Z11069).

\section{Competing Interests}

The authors have declared that no competing interest exists.

\section{References}

1. Chen W, Zheng R, Zeng H, Zhang S, He J. Annual report on status of cancer in China, 2011. Chinese journal of cancer research $=$ Chung-kuo yen cheng yen chiu. 2015; 27: 2-12.

2. Chen W, Zheng R, Zhang S, Zhao P, Zeng H, Zou X, et al. Annual report on status of cancer in China, 2010. Chinese journal of cancer research = Chung-kuo yen cheng yen chiu. 2014; 26: 48-58.

3. Herbst RS, Heymach JV, Lippman SM. Lung cancer. The New England journal of medicine. 2008; 359: 1367-80.

4. van Meerbeeck JP, Fennell DA, De Ruysscher DK. Small-cell lung cancer. Lancet. 2011; 378: 1741-55.

5. Mascaux C, Paesmans M, Berghmans T, Branle F, Lafitte JJ, Lemaitre F, et al. A systematic review of the role of etoposide and cisplatin in the chemotherapy of small cell lung cancer with methodology assessment and meta-analysis. Lung cancer. 2000; 30: 23-36.

6. Pujol JL, Carestia L, Daures JP. Is there a case for cisplatin in the treatment of small-cell lung cancer? A meta-analysis of randomized trials of a cisplatin-containing regimen versus a regimen without this alkylating agent. British journal of cancer. 2000; 83: 8-15.

7. Demedts IK, Vermaelen KY, van Meerbeeck JP. Treatment of extensive-stage small cell lung carcinoma: current status and future prospects. The European respiratory journal. 2010; 35: 202-15.

8. McKeage MJ. Lobaplatin: a new antitumour platinum drug. Expert opinion on investigational drugs. 2001; 10: 119-28.

9. Eisenhauer EA, Therasse P, Bogaerts J, Schwartz LH, Sargent D, Ford R, et al. New response evaluation criteria in solid tumours: revised RECIST guideline (version 1.1). European journal of cancer. 2009; 45: 228-47.

10. Wheate NJ, Walker S, Craig GE, Oun R. The status of platinum anticancer drugs in the clinic and in clinical trials. Dalton transactions. 2010; 39: 8113-27.

11. Ali I, Wani WA, Saleem K, Haque A. Platinum compounds: a hope for future cancer chemotherapy. Anti-cancer agents in medicinal chemistry. 2013; 13: 296-306.

12. Xie CY, Xu YP, Jin W, Lou LG. Antitumor activity of lobaplatin alone or in combination with antitubulin agents in non-small-cell lung cancer. Anti-cancer drugs. 2012; 23: 698-705.

13. Zhou B, Shan H, Zhu KS, Jiang ZB, Guan SH, Meng XC, et al. Chemoembolization with lobaplatin mixed with iodized oil for unresectable recurrent hepatocellular carcinoma after orthotopic liver transplantation. Journal of vascular and interventional radiology: JVIR. 2010; 21: 333-8.

14. Deng QQ, Huang XE, Ye LH, Lu YY, Liang Y, Xiang J. Phase II trial of Loubo(R) (Lobaplatin) and pemetrexed for patients with metastatic breast cancer not responding to anthracycline or taxanes. Asian Pacific journal of cancer prevention: APJCP. 2013; 14: 413-7.

15. Zhang S, Chen J, Yang S, Lin S. An open-label, single-arm phase II clinical study of docetaxel plus lobaplatin for Chinese patients with pulmonary and hepatic metastasis of nasopharyngeal carcinoma. Anti-cancer drugs. 2016; 27 : 685-8.

16. Long GX, Lin JW, Liu DB, Zhou XY, Yuan XL, Hu GY, et al. Single-arm, multi-centre phase II study of lobaplatin combined with docetaxel for recurrent and metastatic nasopharyngeal carcinoma patients. Oral oncology. 2014; 50: 717-20.

17. Nicholson AG, Chansky K, Crowley J, Beyruti R, Kubota K, Turrisi A, et al. The International Association for the Study of Lung Cancer Lung Cancer Staging Project: Proposals for the Revision of the Clinical and Pathologic Staging of Small Cell Lung Cancer in the Forthcoming Eighth Edition of the TNM Classification for Lung Cancer. Journal of thoracic oncology: official publication of the International Association for the Study of Lung Cancer. 2016; 11: 300-11.

18. Roth BJ, Johnson DH, Einhorn LH, Schacter LP, Cherng NC, Cohen HJ, et al. Randomized study of cyclophosphamide, doxorubicin, and vincristine versus etoposide and cisplatin versus alternation of these two regimens in extensive small-cell lung cancer: a phase III trial of the Southeastern Cancer Study Group. Journal of clinical oncology: official journal of the American Society of Clinical Oncology. 1992; 10: 282-91.

19. Noda K, Nishiwaki Y, Kawahara M, Negoro S, Sugiura T, Yokoyama A, et al. Irinotecan plus cisplatin compared with etoposide plus cisplatin for extensive small-cell lung cancer. The New England journal of medicine. 2002; 346: 85-91.

20. Hanna N, Bunn PA, Jr., Langer C, Einhorn L, Guthrie T, Jr., Beck T, et al. Randomized phase III trial comparing irinotecan/cisplatin with etoposide/cisplatin in patients with previously untreated extensive-stage disease small-cell lung cancer. Journal of clinical oncology: official journal of the American Society of Clinical Oncology. 2006; 24: 2038-43.

21. Ying Cheng YF, Xiaoqing Liu et al. A randomized, multicenter phase III study of lobaplatin/etoposide versus cisplatin/etoposide as first-line therapy in patients with extensive-stage small-cell lung cancer and circulating tumor cells (CTCs) as an exploratory biomarker. J Clin Oncol 32:5s, 2014 (suppl; abstr 7595).

22. Welink J, Boven E, Vermorken JB, Gall HE, van der Vijgh WJ. Pharmacokinetics and pharmacodynamics of lobaplatin (D-19466) in patients with advanced solid tumors, including patients with impaired renal of liver function. Clinical cancer research: an official journal of the American Association for Cancer Research. 1999; 5: 2349-58.

23. Chen MQ, Chen C, Lu HJ, Xu BH. The efficacy and toxicities of combined lobaplatin with paclitaxel as a first-line chemotherapy for advanced esophageal squamous cell carcinoma. Journal of thoracic disease. 2015; 7: 1749-55.

24. Huang $Z, X u$ D, Zhang F, Ying $Y$, Song L. Pro-gastrin-releasing peptide and neuron-specific enolase: useful predictors of response to chemotherapy and survival in patients with small cell lung cancer. Clinical \& translational oncology: official publication of the Federation of Spanish Oncology Societies and of the National Cancer Institute of Mexico. 2016; 18: 1019-25.

25. Hirose T, Okuda K, Yamaoka T, Ishida K, Kusumoto S, Sugiyama T, et al. Are levels of pro-gastrin-releasing peptide or neuron-specific enolase at relapse prognostic factors after relapse in patients with small-cell lung cancer? Lung cancer. 2011; 71: 224-8. 